\title{
Pulmonary embolism, spontaneous pneumomediastinum and subcutaneous emphysema in a patient with COVID-19 disease: A case report
}

\author{
Despoina Moumtzi ${ }^{1}$, Anna Gavriilidou ${ }^{1}$, Konstantinos Gasteratos ${ }^{2}$, Dimitrios Vlachakis ${ }^{3}$, Marianna Kakoura ${ }^{1}$
}

\begin{abstract}
The pathophysiology of pulmonary embolism (PE) and pneumomediastinum (PNM) in COVID-19 patients remain unclear. Studies indicate that the infection of the endothelial cells by the virus perpetuates a storm of cytokines and thrombogenic factors, which cause endothelium injury. We present a unique, to our knowledge, case of a patient aged 56 years with COVID-19 pneumonia who was admitted with dyspnea, desaturation, and fever. His situation was complicated by both PNM and PE. He received appropriate treatment with a therapeutic dose of low molecular weight heparin, and exhibited clinical improvement and resolution of the subcutaneous emphysema. Clinicians should suspect both PE and PNM within the differential diagnosis in cases of COVID-19 patients with pleuritic pain, dyspnea, and respiratory failure, after the tenth day from the onset of symptoms.
\end{abstract}

\author{
AFFILIATION \\ 1 Respiratory Department, Papageorgiou \\ General Hospital, Thessaloniki, Greece \\ 2 Plastic and Reconstructive Surgery \\ Department, Papageorgiou General Hospital, \\ Thessaloniki, Greece \\ 3 1st Department of Internal Medicine, \\ Papageorgiou General Hospital, Thessaloniki, \\ Greece
}

\section{CORRESPONDENCE TO}

Despoina Moumtzi. Respiratory Department, Papageorgiou General Hospital, 564 29, Thessaloniki, Greece. E-mail: dmoumtzi@ hotmail.com ORCID ID: https://orcid. org/0000-0001-6967-2313

\section{KEYWORDS \\ COVID-19, pulmonary embolism, pneumomediastinum, subcutaneous emphysema}

Received: 5 September 2021

Revised: 3 October 2021

Accepted: 20 October 2021

\section{INTRODUCTION}

The SARS-CoV-2 pandemic, known as COVID-19, has affected more than 94 million people globally, to date. The risk of thrombosis is increased in these patients. In a United States registry of patients with COVID-19, thrombotic complications occurred in $35.3 \%$ of hospitalized critically-ill patients ${ }^{1}$. However, spontaneous pneumomediastinum (PNM) and subcutaneous emphysema (SE) are rare complications with limited reported cases in the international literature to date?

\section{CASE PRESENTATION}

A man aged 56 years, ex-smoker with an unremarkable medical background, presented to the Emergency Room with a six-day history of fever and dyspnea. The realtime reverse transcription polymerase chain reaction (RTPCR) for SARS-CoV-2 was positive three days before. On physical examination, he had a low-grade temperature of $37.6^{\circ} \mathrm{C}$, tachypnea $(\mathrm{RR}=35 / \mathrm{min})$ and desaturation ( $\mathrm{SpO} 2$ $88 \%$ on $\mathrm{FiO} 2$ 0.21). The rest of his vital signs were as follows: blood pressure of $130 / 70 \mathrm{mmHg}$ and heart rate 85 beats per minute. During auscultation, he had crackles at the base of the lungs bilaterally. Chest $x$-ray showed widespread pulmonary infiltrates (Figure 1a). The baseline laboratory blood tests showed raised inflammatory markers suggestive of acute infection (Table 1). On admission, we used the standard therapy with intravenous azithromycin, and dexamethasone $8 \mathrm{mg} / \mathrm{d}$, subcutaneous prophylactic low molecular weight heparin $(\mathrm{LMWH})$, and supplemental oxygen via nasal cannula (6 L/min).

A chest computed tomography (CT) was performed that showed diffuse ground-glass infiltrations in the basal part of the lower lobes (Figure 2a). On the third hospitalization day, intravenous remdesivir was added to the therapeutic scheme following a decrease in the aspartate aminotransferase (AST) and alanine aminotransferase (ALT) levels (98 IU/L and 112 $\mathrm{IU} / \mathrm{L}$, respectively). His condition deteriorated 3 days later with fever up to $38.5^{\circ} \mathrm{C}$, gradual increase in oxygen demands, and raised inflammatory markers. A new chest x-ray was ordered on the sixth hospitalization day, which revealed cervical subcutaneous emphysema and radiolucent shades parallel to trachea and the left cardiac border. The latter is a characteristic feature of pneumomediastinum, known as 'double wall sign' (Figure 1b). These findings were confirmed by a new chest CT scan (Figure 2b). In addition, it showed interstitial emphysema with a small amount of air around the bronchi and pulmonary vessels in the area of the left hilum due to bronchial or alveolar rupture.

We escalated the oxygen delivery method to a 
Figure 1. a) PA chest $x$-ray on admission with COVID-19 widespread pulmonary infiltrates bilaterally. b) Subcutaneous emphysema (vertical lucent stripes into the neck) and "double bronchial wall sign" suggesting pneumomediastinum. Small line of air parallel to trachea and the left cardiac border

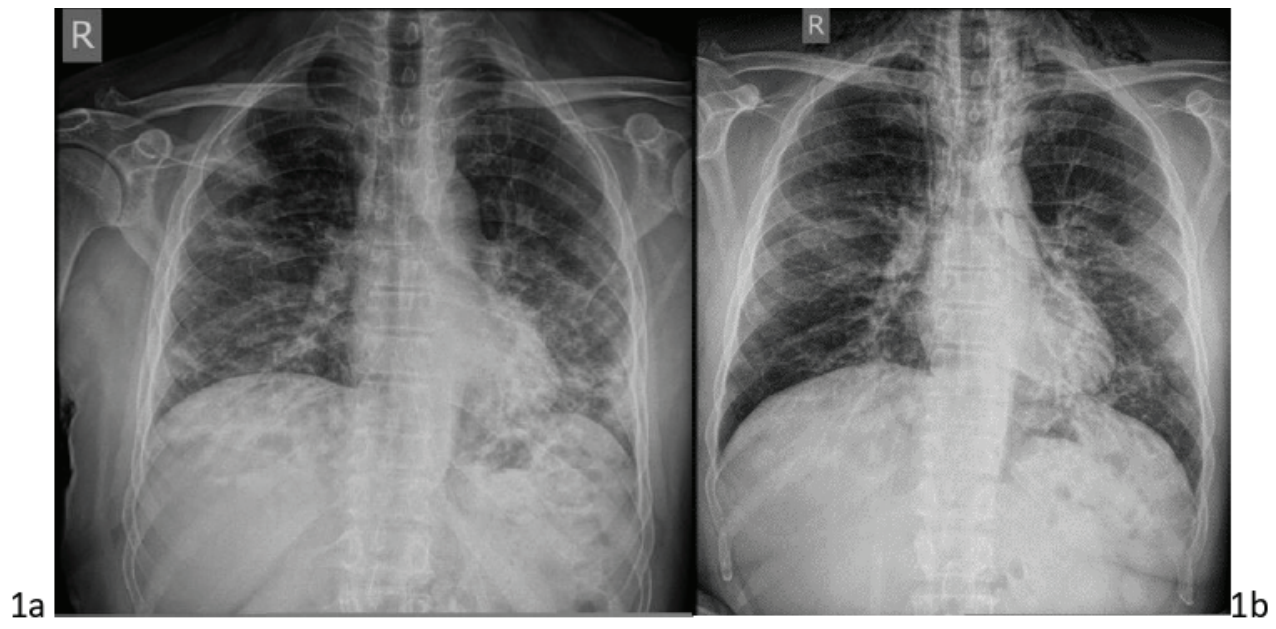

Venturi mask of $\mathrm{FiO} 2$ 0.4, and initiated empirical double intravenous antibiotic therapy with piperacillin/tazobactam and vancomycin to prevent presumed mediastinitis. This antimicrobial treatment was based on the fact that he was already on the sixth day of hospitalization, as well as on the most up-to-date data for COVID-19 coinfections ${ }^{3}$. Therefore, Pseudomonas aeruginosa ${ }^{4}$ and Methicillin-resistant Staphylococcus aureus (MRSA) ${ }^{5}$ had to be covered, while the results from the blood cultures were still pending.

Of note, the patient had no risk factors for development of barotrauma as he did not undergo any procedures (e.g. central venous catheter insertion, or high-flow invasive or non-invasive mechanical ventilation), which would justify his decline. Also, there were no findings of emphysema in the lung parenchyma, such as subpleural bullae, which would theoretically rupture. In the following monitoring period, the SE was marked to the neck and the assessment of its progression was made by the presence of crepitus on palpation.

On the eleventh day of hospitalization, the patient complained of shortness of breath associated with pain in the left calf. A significant increase $(\times 10)$ in d-dimers value of

Table 1. Laboratory markers on admission and throughout hospitalization

\begin{tabular}{|c|c|c|c|c|c|}
\hline Laboratory results & Admission & Day 1 & Day 3 & Day 6 & $\begin{array}{c}\text { Day } 28 \\
\text { discharge }\end{array}$ \\
\hline C-reactive protein (CRP) NR $<0.8 \mathrm{mg} / \mathrm{dL}$ & 7.55 & 6.72 & 10.3 & & 1.43 \\
\hline Procalcitonin $(\mathrm{PCT}) \quad \mathrm{NV}<0.05 \mathrm{ng} / \mathrm{mL}$ & 0.27 & 0.16 & 0.27 & 0.17 & 0.05 \\
\hline White cell count $N R=(3.9-11.1) \times 10^{3} / \mu \mathrm{L}$ & 9.86 & 10.8 & 10.1 & 8.9 & 8.38 \\
\hline Neutrophils (\%) & 79.9 & 93 & 92.7 & 81.9 & \\
\hline Lymphocytes (\%) & 15.6 & 4.8 & 5.5 & 4.7 & 22.4 \\
\hline Platelets $N R=(150-400) \times 10^{3} / \mu \mathrm{L}$ & 134 & 108 & 100 & & \\
\hline Hematocrit (\%) & 46.6 & 44.6 & 44.8 & 42.4 & 42.8 \\
\hline Hemoglobin (g/dL) & 15.8 & 15.3 & 15.2 & 14.5 & 14.6 \\
\hline Ferritin NR=4.6-204 ng/mL & 1088 & & 2373 & 1622 & 1180 \\
\hline D-dimers $\mathrm{NV}<500 \mathrm{ng} / \mathrm{mL}$ & 1450 & 6780 & 23120 & 37730 & 3680 \\
\hline Fibrinogen $\mathrm{NR}=200-400 \mathrm{mg} / \mathrm{dL}$ & 548 & 555 & 532 & & 593 \\
\hline Lactate dehydrogenase $(\mathrm{LDH}) \mathrm{NR}=200-400 \mathrm{IU} / \mathrm{L}$ & 907 & 1604 & 720 & 592 & 350 \\
\hline Aspartate aminotransferase $(A S T) N R=10-40 \mathrm{IU} / \mathrm{L}$ & 173 & 126 & 98 & 94 & 78 \\
\hline Alanine aminotransferase $(A L T) N R=10-55 \mathrm{IU} / \mathrm{L}$ & 197 & 158 & 112 & 98 & 87 \\
\hline
\end{tabular}


Figure 2. a) CT on day 3 admission with typical ground-glass pulmonary infiltrations suggestive of COVID pneumonia primarily affecting the basal parts of both lungs. b) CT showing widespread subcutaneous cervical emphysema around the strap muscles, and around the trachea in the superior mediastinum. Signs are suggestive of pneumomediastinum. c) CTPA showing pulmonary embolism in peripheral segmental branches of the pulmonary artery at different levels of cross sections (silver arrows)
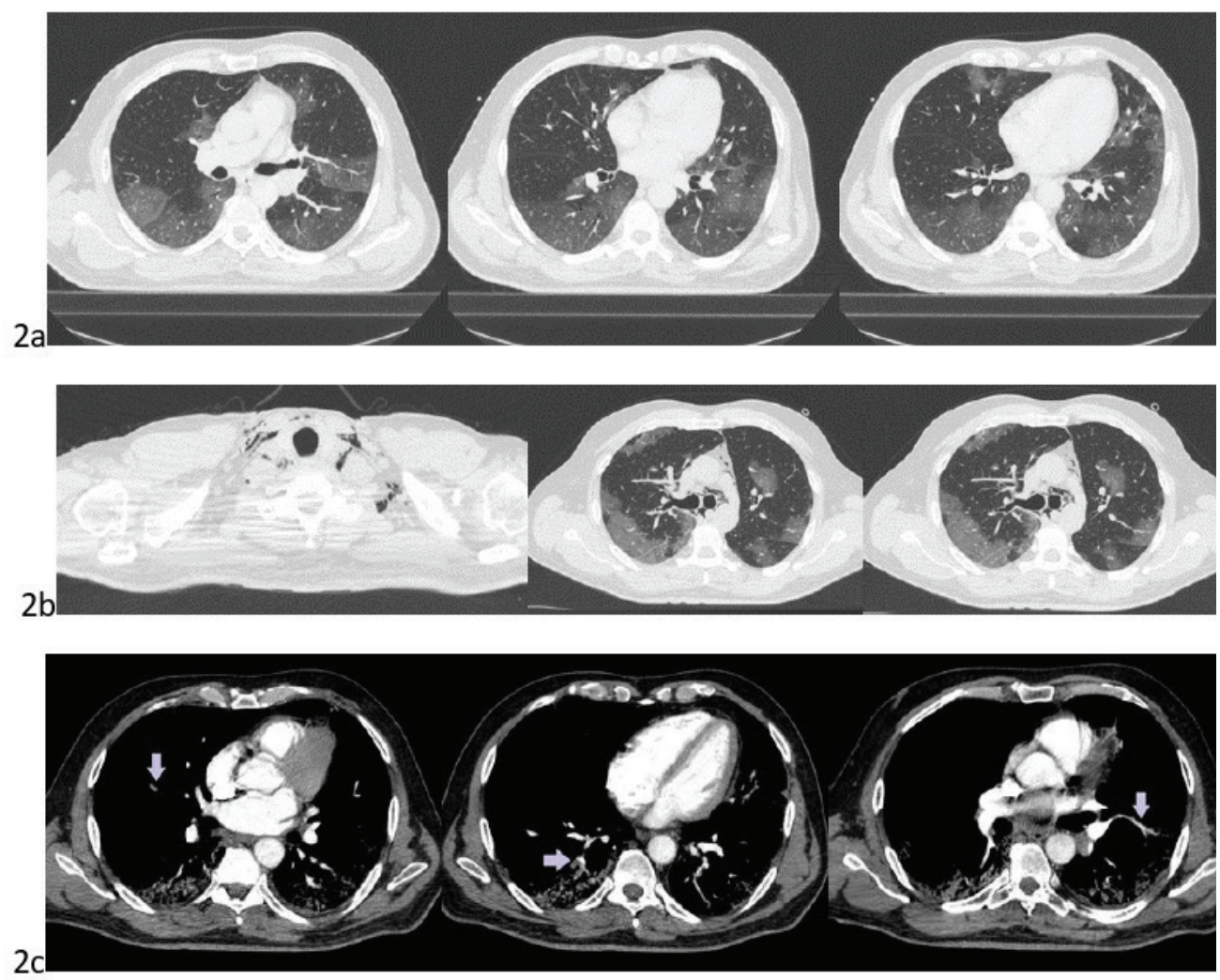

36000 was noted (normal range $<500 \mu \mathrm{g} / \mathrm{L}$ ). The patient's oxygen demands were elevated, the ECG revealed sinus rhythm and tachycardia while the SE had almost completely resolved clinically. A computed tomography pulmonary angiography (CTPA) confirmed the diagnosis of pulmonary embolism (PE); filling defects were observed in the segmental and subsegmental branches of the pulmonary artery (PA) supplying the middle and lower lobes bilaterally (Figure 2c). We emphasize that the SE and the presence of free air in the anterior mediastinum had resolved within 5 days. However, the ground-glass infiltrations worsened with localized consolidations in the lungs. The patient was immediately commenced on a therapeutic dose of LMWH (tinzaparine $175 \mathrm{iu} / \mathrm{kg}$ OD: $175 \times 80 \mathrm{~kg}=14000 \mathrm{iu} / \mathrm{d}$ ).

Within the following days, there was a remarkable overall clinical improvement as his oxygen demands became minimal, there were no spikes of fever and he remained hemodynamically stable during the rest of his hospitalization. He was discharged home with instructions to continue a course of therapeutic LMWH. An outpatient follow-up appointment was scheduled to evaluate his clinical progress in a month.

\section{DISCUSSION}

Pneumomediastinum is a rare complication of pneumonia due to COVID-19. There are two categories: primary (or spontaneous), and secondary (or traumatic), which may result from medical interventions ${ }^{6,7}$. Men are more often affected than women, with dyspnea and pleuritic chest pain being the most common presenting symptoms. Clinical signs include tachycardia, Hamman's sign, ECG changes (i.e. premature contractions, inverted T-waves, low voltage readings), and rarely tension physiology (tension PNM) from direct cardiac and lung compression?. Chest radiograph and CT are the appropriate diagnostic tools for pneumomediastinum. If missed, more severe complications like pneumopericardium may result causing tamponade and mediastinitis ${ }^{7}$. Early diagnosis is essential for a proper treatment as the mortality is high.

Factors predisposing to air leak include alveolar rupture secondary to trauma or differences in pressure gradient, such as in cough, vomiting and other Valsava maneuvers, as well as inflammatory processes of the alveolar wall itself. Many researchers advocate that the pathophysiology 
of pneumomediastinum in COVID-19 patients is related to diffuse alveolar damage (DAD), cellular fibromyositis exudates, evident desquamation of pneumocytes and hyaline membrane formation ${ }^{2,6}$. As a result, gas leaks into the pulmonary interstitium, therefore causing interstitial emphysema ${ }^{6}$. The same findings were noticed in the CT scan of our patient. Pneumomediastinum may also be associated with pneumopericardium, and/or pneumothorax (PTX) 2,6 .

It is postulated that the virus disrupts the vascular endothelium by direct viral penetration into the endothelial cells in a similar manner as it does in the alveoli. Thus, it is believed that the PE as a complication in COVID-19 patients results from endothelial injury, which in turn releases cytokines and von Willebrand factor $(\mathrm{VWF})^{8}$. These proinflammatory cytokines promote vascular endothelial cell apoptosis, pulmonary microthrombosis, vascular leakage and alveolar edema ${ }^{9}$. In addition, cytokine storm, macrophages, thrombin, platelets, monocytes, lymphocytes, and VWF, contribute to a hypercoagulable state in COVID-19. Lastly, hypoxia predisposes to thrombosis due to blood hyperviscosity ${ }^{10}$.

Despite the administration of prophylactic antithrombotic treatment in COVID-19 patients, the incidence of pulmonary embolism (PE) has been reported twice as high, especially in the patients admitted in the intensive care unit for supportive care due to COVID-19 pneumonia ${ }^{11}$. A systematic review by Liao et al. ${ }^{12}$ reported that nearly 2 in 10 patients developed PE from a total of 1835 COVID-19 patients. Preliminary studies in COVID-19 cohorts support that the prevalence of PE in these patients may range from 23 to 30\%. The same studies showed that contrast-enhanced computed tomography pulmonary angiography (CTPA) is the gold standard for the diagnosis of $\mathrm{PE}^{13,14}$.

To the best of our knowledge, there are no published COVID-19 cases complicated by PE with concomitant pneumomediastinum. Perhaps, undetectable microemboli in peripheral branches of the PA occurred ${ }^{14}$. However, there are published COVID-19 cases with merely PNM ( $n=6 ; 66 \%$ males), and other cases with a combination of PNM and PTX $(n=5)^{15}$. Radiological findings of subcutaneous emphysema were present in three out of the six patients with PNM, and in two out of the five patients with PNM/PTX ${ }^{4}$ Similarly, to our case, no specific treatment was required since the symptoms were self-limited within a short period of time. The outcome was favorable in $50 \%$ of the patients. However, $22.2 \%$ of the patients who passed away suffered from severe comorbidities ${ }^{4}$.

It is speculated that the prognosis in patients with PNM depends on the extent of the pulmonary injury ${ }^{2}$. We noticed in this particular case that the development of the pneumomediastinum coincided with the deterioration of preexisting ground-glass infiltrations and localized 'crazy paving' pattern in the lower lobes, which did not pre-exist. Also, an elevated lactate dehydrogenase $(\mathrm{LDH})$ is possibly a risk factor with prognostic value for patients with $\mathrm{PNM}^{2,6}$. Similarly, an elevated LDH (mean $863 \mathrm{U} / \mathrm{L}$ ) was associated with poorer prognosis in SARS patients. In our case, the development of pneumomediastinum coincided with a doubling of the $\mathrm{LDH}$ value from 500 to $1000 \mathrm{U} / \mathrm{L}$, and subsequent gradual decrease. The appearance of PNM was clinically subtle on the 12th day from the onset of symptoms, and compared to other cases, it was rather of early onset as usually it may occur late, between the 18th and 27 th day of symptoms ${ }^{6}$.

\section{CONCLUSION}

Clinicians should suspect both PE and PNM within the differential diagnosis in cases of COVID-19 patients with pleuritic pain, dyspnea, and respiratory failure, after the tenth day from the onset of the symptoms. The simultaneous presence of these conditions in a patient may be lifethreatening in an already compromised patient due to COVID-19 pneumonia. Clinicians must become aware of this novel presentation and initiate the proper treatment immediately.

\section{CONFLICTS OF INTEREST}

The authors have completed and submitted the ICMJE Form for Disclosure of Potential Conflicts of Interest and none was reported.

\section{FUNDING}

There was no source of funding for this research.

\section{ETHICAL APPROVAL AND INFORMED CONSENT}

Ethical approval was not required for this study. The patient provided informed consent for the publication of this report and any accompanying images.

\section{DATA AVAILABILITY}

The data supporting this research cannot be made available for privacy reasons.

\section{PROVENANCE AND PEER REVIEW}

Not commissioned; externally peer reviewed.

\section{REFERENCES}

1. Piazza G, Morrow DA .Diagnosis, Management, and Pathophysiology of Arterial and Venous Thrombosis in COVID-19. JAMA. 2020;324(24):2548-2549. doi:10.1001/jama.2020.23422

2. Shan S, Guangming L, Wei L, et.al. Spontaneous pneumomediastinum, pneumothorax and subcutaneous emphysema in COVID-19: case report and literature review. Rev Inst Med Trop Sao Paulo. 2020;62:e76. doi:10.1590/s1678-9946202062076

3. Hughes S, Troise O, Donaldson $\mathrm{H}$, et.al. Bacterial and fungal coinfection among hospitalized patients with COVID-19: a retrospective cohort study in a UK secondary-care setting. Clin Microbiol Infect. 2020;26(10):1395-1399. 
doi:10.1016/j.cmi.2020.06.025

4. Restrepo MI, Babu BL, Reyes LF, et.al. Burden and risk factors for Pseudomonas aeruginosa community-acquired pneumonia: a multinational point prevalence study of hospitalised patients. Eur Respir J. 2018;52(2):1701190. doi:10.1183/13993003.01190-2017

5. Bouchiat C, Curtis S, Spiliopoulou I, et.al. MRSA infections among patients in the emergency department: a European multicenter study. J Antimicrob Chemother. 2017;72(2):372375. doi:10.1093/jac/dkw431

6. Hazariwala V, Hadid H, Kirsch D, et.al. Spontaneous pneumomediastinum, pneumopericardium, pneumothorax and subcutaneous emphysema in patients with COVID-19 pneumonia, a case report. J Cardiothorac Surg. 2020;15(1):301. doi:10.1186/s13019-020-01308-7

7. Wolfe MW, Meltzer JS. Delayed Tension Pneumomediastinum after Cardiac Surgery. Anesthesiology. 2018;129(4):809. doi:10.1097/ALN.0000000000002257

8. Sakr Y, Giovini M, Leone M. Pulmonary embolism in patients with coronavirus disease-2019 (COVID-19) pneumonia: a narrative review. Ann Intensive Care 2020;10(1):124. doi:10.1186/s13613-020-00741-0

9. De Lorenzo A, Escobar S, Tibiriçá E. Systemic endothelial dysfunction: A common pathway for COVID-19, cardiovascular and metabolic diseases. Nutr Metab Cardiovasc Dis. 2020;30(8):1401-1402. doi:10.1016/j.numecd.2020.05.007

10. Oudkerk M, Büller HR, Kuijpers D, et.al. Diagnosis, Prevention, and Treatment of Thromboembolic Complications in COVID-19: Report of the National Institute for Public Health of the Netherlands. Radiology. 2020;297(1):E216-E222. doi:10.1148/radiol.2020201629

11. Poissy J, Goutay J, Caplan M, et al. Pulmonary Embolism in Patients With COVID-19: Awareness of an Increased Prevalence. Circulation. 2020;142(2):184-186. doi:10.1161/CIRCULATIONAHA.120.047430

12. Liao SC, Shao SC, Chen YT, et al. Incidence and mortality of pulmonary embolism in COVID-19: a systematic review and meta-analysis. Crit Care. 2020;24(1):464. doi:10.1186/s13054-020-03175-z

13. Grillet F, Behr J, Calame P, et al. Acute Pulmonary Embolism Associated with COVID-19 Pneumonia Detected with Pulmonary CT Angiography. Radiology. 2020;296(3):E186-E188. doi:10.1148/radiol.2020201544

14. Léonard-Lorant I, Delabranche X, Séverac F, et al. Acute Pulmonary Embolism in Patients with COVID-19 at CT Angiography and Relationship to d-Dimer Levels. Radiology. 2020;296(3):E189-E191. doi:10.1148/radiol.2020201561

15. Thetford J, Kahn M, Barot N, et al. Pneumomediastinum and pneumothorax due to noninvasive positive pressure ventilation in covid-19 pneumonia. Chest. 2020;158(4):A1284. doi:10.1016/j.chest.2020.08.1171 